BULL. AUSTRAL. MATH. SOC.

$49 A 27,49 A 10$

VOL. 27 (1983), 395-401.

\title{
ON THE EXISTENCE OF OPTIMAL CONTROLS \\ IN BANACH SPACES
}

\author{
Mohan Joshi
}

We prove the existence of an optimal control in Banach spaces for a system characterized by Hammerstein operator equations.

\section{Introduction}

Let $X$ be a Banach space with dual $X^{*}$ and $Y \subset X^{*}$ a closed subspace. Let $Z$ be another Banach space and $U \subset Z$ be a weakly compact set. We consider a system characterized by the operator equation

$$
x+K_{u}^{N x}=w
$$

where $u \in U$ is a control variable and for each $u \in U, K_{u}: X \rightarrow X^{*}$ is a linear operator with range in $Y$ and $N: Y \rightarrow X$ a nonlinear operator. $w \in X^{*}$ is given. For a fixed $u$, the operator equation (1.1) is called the Harmerstein operator equation. Existence and uniqueness of solutions for such types of equations have been studied by many authors (refer Browder [2]).

Let $\phi$ be a lower semicontinuous functional on $X^{*}$ with values in $R^{+}$and $S$ a closed and bounded subset of $X$. The problem is to find a control $u^{*} \in U$ such that

$$
J(u)=\phi(x)
$$

is minimum, subject to the constraint that $x \in S$ is the response of the system (1.1) corresponding to the control $u$.

Received 2 February 1983. 
In this paper we prove existence of an optimal control under continuity assumptions on $\left\{K_{u}\right\}_{u \in U}$ and some mild assumptions on $N$. As a corollary we derive the existence of a control function $u^{*}$ for an important.system characterized by the equation

$$
x(t)+\int_{0}^{t} f(\tau, x(\tau)) u(\tau) d \tau=x_{0}
$$

with the associated functional $J(u)$ given by

$$
J(u)=\phi(x, u)=\int_{0}^{T} g(t, x(t), u(t)) d t,
$$

where $T \in[0, \infty)$ is prescribed.

\section{Main results}

LEMMA 2.1. Let $X$ be a real reflexive Banach space with dual $X^{*}$ and $Y \subset X^{*}$ a closed subspace. Let $Z$ be conother Banach space and $s \subset X^{*}$ be a bounded set. Let, for $u \in Z, K_{u}: X \rightarrow X$ be a bounded Zinear operator with range in $Y$ and $N: Y \rightarrow X$ a continuous and bounded nonlinear operator. Further assume that the following hold:

(a) $K_{u}$ is compact for each $u \in 2$;

(b) $u_{n} \rightarrow u$ in $z$ implies that $K_{u_{n}} \rightarrow K_{u}$ in operator norm.

Let $\left\{u_{n}\right\}$ be any sequence in $z$ which converges weakly to $u^{*}$ in $z$ and let $x_{n} \in S$ denote a solution of (1.1) corresponding to $u_{n}$. Then there exists a subsequence $\left\{x_{n_{k}}\right\}$ of $\left\{x_{n}\right\}$ which converges to $x^{*}$ and $x^{*}$ is a solution of (1.1) corresponding to $u^{*}$.

Proof. Since $x_{n} \in S$ is a solution of (1.1) corresponding to $u_{n}$, we have

$$
x_{n}+K_{u_{n}} N x_{n}=w
$$

$\left\{x_{n}\right\}$ is a bounded sequence in a reflexive Banach space and hence there 
exists a subsequence $\left\{x_{n_{k}}\right\}$ of it which converges to $x^{*}$ weakly.

Similarly boundedness of $N$ implies that there exists a subsequence of $\left\{N x_{n_{k}}\right\}$ (which we again denoted by $\left\{N x_{n_{k}}\right\}$ ) converging to $y$ weakly.

Since $K_{u_{k}} \rightarrow K_{u^{*}}$ in operator norm and $\left\{N x_{n_{k}}\right\}$ is bounded, it follows that

$$
K_{u_{k}} N x_{n_{k}}-K_{u^{*}} N x_{n_{k}} \rightarrow 0 \text { as } k \rightarrow \infty \text {. }
$$

Also, since $N x_{n_{k}} \rightarrow y$ and $K_{u^{*}}$ is compact, we have

$$
K_{u^{*}} x_{n_{k}} \rightarrow K_{u^{*}}
$$

Combining (2.2) and (2.3) we get that $K_{u_{n_{k}}} N x_{n_{k}} \rightarrow K_{u^{*}} y$ as $k \rightarrow \infty$. But

(2.1) gives

$$
x_{n_{k}}=w-K_{u_{k}} N x_{n_{k}}
$$

and hence $\left\{x_{n_{k}}\right\}$ is strongly convergent, that is $x_{n_{k}} \rightarrow x^{*}$. Now continuity of $N$ implies that $N x_{n_{k}} \rightarrow N x^{*}$ and hence $K_{u_{n_{k}}} N x_{k} \rightarrow K_{u^{*}} N x^{*}$. So (2.4) gives

$$
x^{*}+K_{u^{*}}^{N x^{*}}=w
$$

That is $\left\{x_{n_{k}}\right\}$ converges strongly to $x^{*}$ where $x^{*}$ is a solution of the system (1.1) corresponding to $u^{*}$.

DEFINITION 2.1. Let the spaces $X, Y, Z$ be as in the above lemma. Let $U \subset Z$ be a weakly compact subset. $X=\left\{x_{u}: u \in U\right\}$ is said to be the set of trajectories of (1.1) if

$$
\begin{aligned}
& \text { (i) } X \neq \varnothing, \\
& \text { (ii) } x_{u} \in Y \text {, }
\end{aligned}
$$


(iii) $x_{u}$ satisfies the operator equation

$$
x_{u}+K_{u} N x_{u}=w
$$

DEFINITION 2.2. $F \subset Y$ is said to be an attainable set of the system (1.1) if $F=\{y: y=x$ for some $x$ in $X\}$.

THEOREM 2.1. Let $F$ be an attainable set of the system (1.1) and $S$ a closed and bounded subset of $y$ such that $F \cap S \neq \emptyset$. Let $\phi$ be a Zower semicontinuous functional on $X^{*}$ with values in $R^{+}$. Then there exists $u^{*} \in U$ where it attains a minimum to the functional $\phi$ on the set $F \cap S$.

Proof. By Lemma 2.1, $F \cap S$ is a compact set. Since $\phi$ is a lower semicontinuous functional and $F \cap S$ is compact, it follows that $\phi$ attains its minimum on $F \cap S$. Hence the result.

As a corollary of the above theorem we obtain the existence of an optimal control for the system characterized by the equation

$$
x(t)+\int_{0}^{t} f(\tau, x(\tau)) u(\tau) d \tau=x_{0},
$$

where the control function $u(t)$ lies in some weakly compact subset of $L_{2}^{m}[0, T], 0 \leq T<\infty$. The associated cost functional $J(u)$ to be minimized is given by

$$
J(u)=\phi(x)=\int_{0}^{T} g(\tau, x(\tau), u(\tau)) d \tau .
$$

ASSUMPTION [A]. $f(t, x): R \times R^{n} \rightarrow R^{n \times m}$ is such that

[Al] $f(t, x)$ is measurable in $t$ for all $x \in R^{n}$,

[A2] $f(t, x)$ is continuous in $x$ for almost all $t \in[0, T]$,

[A3] $\|f(t, x)\| \leq\|a(t)\|+b\|x\|, a(t) \in L_{2}^{n \times m}[0, T], b>0$ for all $(t, x) \in R \times R^{n}$.

Here the norm in the left hand side denotes the $k^{n \times m}$ norm and the norm in the right hand side denotes the $R^{n}$ norm. 
ASSUMPTION [B]. $g(t, x, u): R \times k^{n} \times R^{m} \rightarrow R$ is such that

[BI] $g(t, x, u)$ is measurable in $t$ for all $(x, u) \in R^{n} \times R^{m}$,

[B2] $g(t, x, u)$ is continuous in $(x, u) \in R^{n} \times R^{m}$ for almost all $t \in[0, T]$,

[B3] there exists $\Psi \in L_{1}[0, T]$ such that $g(t, x, u) \geq \Psi(t)$ for almost all $t \in[0, T]$ and all $(x, u) \in R^{n} \times R^{m}$,

[B4] $g(t, x, u)$ is convex in $u$ for all $t, x$.

We set $X=L_{2}^{n \times m}[0, T], Y=L_{2}^{n}[0, T], Z=L_{2}^{m}[0, T]$. For each $u \in Z$, define $K_{u}: X \rightarrow X$ with range in $Y$ as follows:

$$
\left[K_{u} x\right](t)=\int_{0}^{t} x(\tau) u(\tau) d \tau
$$

$N: Y \rightarrow X$ is defined as

$$
[N x](t)=f(t, x(t)) .
$$

With these definitions, (2.5) is equivalent to the operator equation

$$
x+K_{u} N x=x_{0} .
$$

LEMMA 2.2. $K_{u}$ is a bounded linear operator from $X$ into itself with range $Y$ such that

(a) $k_{u}$ is compact for each $u \in Z$,

(b) $u_{n}-u$ in 2 implies that $K_{u_{n}} \rightarrow K_{u}$.

Proof. $\left[K_{u} x\right](t)=\int_{0}^{t} x(\tau) u(\tau) d \tau$, where $x \in L_{2}^{n \times m}[0, T]$, $u \in L_{2}^{m}[0, T] . \quad$ Let

$$
K(t, \tau)= \begin{cases}0, & t \leq \tau, \\ I, & \tau<t .\end{cases}
$$

Then we get 


$$
[K u x](t)=\int_{0}^{T}[K(t, \tau) x(\tau)] u(\tau) d \tau
$$

Since ess $\sup \int_{0}^{T}\|K(t, \tau)\|^{2}\|u(\tau)\|^{2} d \tau<\infty$ it follows by the theory of integral operators (refer Okikiolu [3]) that $K_{u}$ is compact for each $u \in L_{2}^{m}[0, T]$. Similarly one can show that $K_{u}$ is compact with respect to the variable $u$ and hence by using the uniform boundedness principle we get the result.

LEMMA 2.3. Under Assumption [A] the non linear operator $N$ is a continuous and bounded operator from $L_{2}^{n}[0, T]$ to $L_{2}^{n \times m}[0, T]$.

COROLLARY. Let $f$ and $g$ satisfy Assumptions [A] and [B] respectively. Let $U$ be a weakly compact subset of $L_{2}^{m}[0, T]$. For $u \in U$, let (2.5) possess a solution in a closed and bounded set $S \subset L_{2}^{n}[0, T]$. Then there exists $u^{*} \in U$ such that

$$
J\left(u^{*}\right)=\inf J(u)
$$

where $J(u)$ is the cost functional given by (2.6).

Proof. We set $X=L_{2}^{n \times m}, Y=L_{2}^{n}[0, T], Z=L_{2}^{m}[0, T]$ and $K_{u}$ and $N$ be as defined before. Then (2.5) is equivalent to the operator equation

$$
x+K_{u} N x=x_{0} \text {. }
$$

Let $F$ denote the attainable set of (2.5). Then by assumption $F \cap S \neq \emptyset$. Further, by a result of Berkovitz [1], $\phi$ is lower semicontinuous with respect to weak convergence in $u$ and strong convergence in $x$. Since all the conditions of Theorem 2.1 are satisfied, it follows that there exist $u^{*} \in U$ such that

$$
J\left(u^{*}\right)=\inf _{u \in U} J(u)
$$

REMARK. Vidyasagar [4] has proved a similar result for the system (2.5). However he imposes a Lipschitz condition on $f(t, x)$ assume a simple growth condition of type [A3]. This is a significant 
improvement.

\section{References}

[1] Leonard D. Berkovitz, "Lower semicontinuity of integral functionals", Trons. Amer. Math. Soc. 192 (1974), 51-57.

[2] Felix E. Browder, "Nonlinear functional analysis and nonlinear integral equations of Hammerstein and Urysohn type", Contributions to nonlinear functional analysis, 425-500 (Academic Press, New York, London, 1971).

[3] 6.0. Okikiolu, Aspects of the theory of bounded integral operators in $L^{p}$-spaces (Academic Press, London, New York, 1971).

[4] M. Vidyasagar, "On the existence of optimal controls", J. Optim. Theory App I. 17 (1975), 273-278.

\footnotetext{
Mathematics Group,

BITS,

Pilani 333031 ,

India.
} 